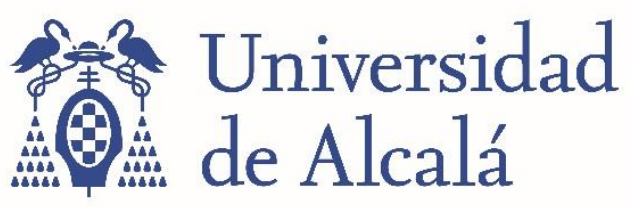

BIBLIOTECA

Document downloaded from the institutional repository of the University of Alcala: http://dspace.uah.es/

This is a postprint version of the following published document:

Angulo-Vinuesa, X., Bravo, M., Martín-López, S., López-Amo, M., GonzálezHerrráez, M., "Wide range group delay tuning in lossy fiber ring resonators" Proc. SPIE 8421, 842197, (2012)

Available at http://dx.doi.org/10.1117/12.974947

Copyright 2009 Society of Photo-Optical Instrumentation Engineers. One print or electronic copy may be made for personal use only. Systematic electronic or print reproduction and distribution, duplication of any material in this paper for a fee or for commercial purposes, or modification of the content of the paper are prohibited.

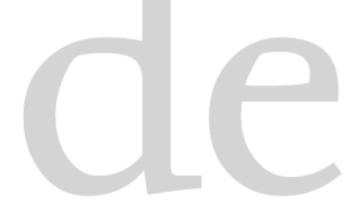

(Article begins on next page)

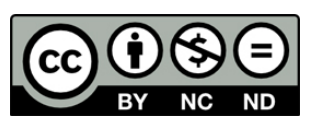

This work is licensed under a

Creative Commons Attribution-NonCommercial-NoDerivatives

4.0 International License. 


\title{
Wide range group delay tuning in lossy fiber ring resonators
}

\author{
Xabier Angulo-Vinuesa*a ${ }^{\mathrm{a}}$, Mikel Bravo ${ }^{\mathrm{b}}$, Sonia Martin-Lopez ${ }^{\mathrm{a}}$, Manuel Lopez-Amo ${ }^{\mathrm{b}}$ and Miguel \\ Gonzalez-Herraez ${ }^{\mathrm{c}}$ \\ ${ }^{a}$ Instituto de Óptica - Consejo Superior de Investigaciones Científicas, Madrid (Spain); \\ ${ }^{\mathrm{b}}$ Departamento de Ingeniería Eléctrica y Electrónica, Universidad Pública de Navarra, Pamplona \\ (Spain); ${ }^{c}$ Departamento de Electrónica - Escuela Politécnica, Universidad de Alcalá, Alcalá de \\ Henares (Spain)
}

\begin{abstract}
We demonstrate theoretically and experimentally that a wide-range tuning of group delay values can be achieved in a lossy fiber ring resonator. The tuning mechanism relies simply on varying the loss/coupling ratio in the resonator. This simple structure may be used advantageously in different regimes for many sensing configurations, both for achieving extremely high sensitivity enhancements (by working close to critical coupling, where the group index becomes extremely large) or suppression of undesired refractive index effects (e.g. Kerr effect), by working in the under-coupled regime.
\end{abstract}

Keywords: slow light, ring resonators, group delay, group index, Kerr effect.

\section{INTRODUCTION}

Light-matter interactions are weak in most optical media in conventional conditions. Slow light structures allow a large reduction of the group velocity of the light signals travelling through them, and, as a consequence, a strong confinement of the electromagnetic field and a great enhancement of light-matter interactions [1]. These interactions include various linear and nonlinear effects as well as spontaneous emission.

From the point of view of fiber sensors, the possibility of enhancing light-matter interactions may favor the development of more compact and sensitive devices. Some research effort has been devoted to understanding the exact enhancement values given by slow light in different light-matter interactions. For instance, the role of slow light in enhancing nonlinear effects has been theoretically investigated $[2,3]$. The enhancement factor in Kerr effect scales as $n_{g}{ }^{2}\left(n_{g}\right.$ being the group index of the structure) due to the combined effect of two contributions: the longer transient time of light pulses in the medium and the higher energy density due to spatial pulse compression. It has also been theoretically and experimentally proved that the extreme dispersion of slow light can lead to an enhancement in the spectral sensitivity of interferometers given by $n_{g}$ [4]. The role of slow light in enhancing gyroscope performance [5] and Beer-LambertBouguer (BLB) absorption [6,7] has also been theoretically and/or experimentally investigated. Generally speaking, light-matter interactions have been found to be only enhanced in structural slow light systems (e.g. coupled resonators, Bragg gratings, etc.). Material slow light systems (e.g. stimulated Brillouin/Raman scattering, parametric amplification, etc.) do not follow the same rules, the origin of this difference being that the electromagnetic energy velocity in material slow light does not depend on the group index [8]. From the fiber sensing point of view, it is therefore interesting to find structural slow light media, i.e. passive structures in which the group index could be tuned widely. Such a system could be considered as a platform for the development of slow-light assisted sensors. In this paper we demonstrate theoretically and experimentally a wide range tuning of the group delay in a lossy fiber-based ring resonator. We believe that this structure could become a basic building block in future slow-light-assisted fiber sensors.

\section{THEORETICAL ANALYSIS}

In this section we perform a theoretical study of slow and fast light in lossy fiber ring resonators. We show that, depending on the coupling ratio and the loss in the resonator, the group delay of the ring resonator can be tuned from strong delay to strong advancement, including situations of negative group delay. 




Fig.1: Fiber-based ring resonator considered. Light travels from left to right.

We consider the structure depicted in Figure 1, i.e. the typical fiber ring resonator with a variable loss element inside. Our analysis of this structure is similar to the analysis done by Heebner et al. [9] for resonator-coupled waveguides. A transfer function can be found for the case of the lossy resonator:

$$
\frac{E_{o}}{E_{i}}=\frac{\kappa \exp \left(\frac{i \omega n L}{c}\right)-a}{\exp \left(\frac{i \omega n L}{c}\right)-\kappa a}
$$

Where $\kappa$ and $a$ are, respectively, the coupling ratio and the attenuation in the resonator (in unitless form). As expected, this transfer function leads to resonances in the spectrum with a periodicity given by $c / n L$. These resonances exhibit steep dispersion slopes close to the center, leading to large group delays. We can theoretically evaluate the group delay in the resonances by finding the phase of expression (1), obtaining the derivative with respect to $\omega$, and evaluating the obtained expression at the resonant frequencies. The resulting expression is:

$$
\tau_{g}=\frac{n L}{c} \cdot \frac{a\left(1-\kappa^{2}\right)}{\kappa\left(a^{2}+1\right)-a\left(\kappa^{2}+1\right)}
$$

We can therefore see that the delay in the resonator can be expressed as the regular single-pass delay of the resonator $(n L / c)$ multiplied by a factor that may be varied continuously by changing only the attenuation and/or coupling ratio in the resonator. It can be easily shown that, when the losses exceed the coupling (undercoupling), negative group delays can be found at the resonances, while they remain positive in the opposite case (overcoupling). A discontinuity in function (2) is found for the critical coupling case $(a=\kappa)$, where the group delay function displays a vertical asymptote, taking values of $-\infty$ when $a \rightarrow \kappa^{-}$and $+\infty$ when $a \rightarrow \kappa^{+}$. In the transmission coefficient, the case $a=\kappa$ (critical coupling) corresponds to the case in which the transmission at the resonance center goes to zero. The qualitatively different behavior of both regimes is depicted in Fig. 2 .
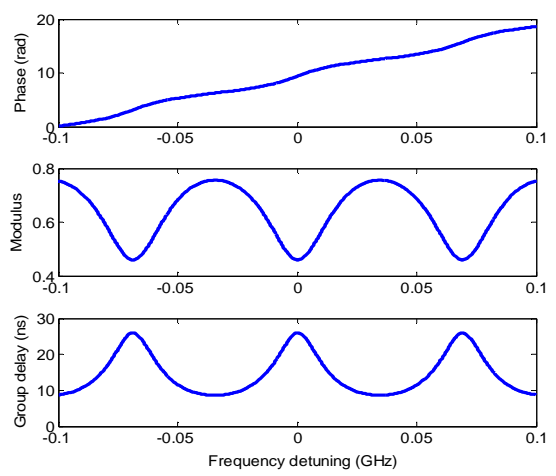

(a)
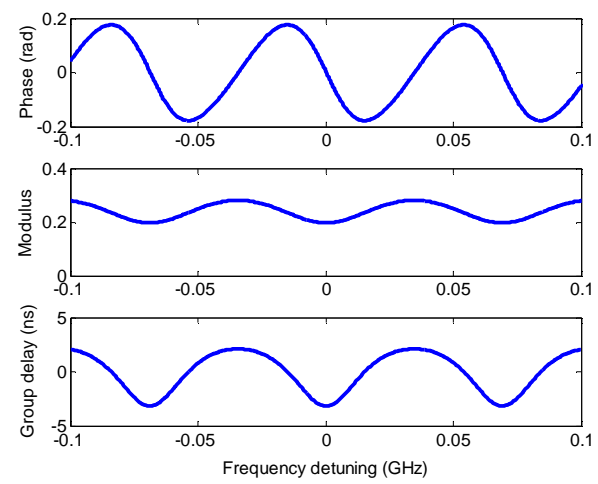

(b)

Fig. 2: Phase, modulus and group delay response vs. frequency for different attenuation values within a 3 meter fiber resonator, $\kappa=0.24$ : (a) $2 \mathrm{~dB}$ attenuation in the loop; (b) $13.5 \mathrm{~dB}$ attenuation in the loop. 


\section{EXPERIMENTS}

To confirm the previous theoretical analysis, we developed a simple experiment. We constructed the structure of Figure 3 with a variable fiber coupler and a precision optical attenuator in the loop. The total length of fiber in the loop amounts $\sim 3$ meters. An Optical Vector Analyzer (OVA) was used to measure the group delay response of the structure. Since the OVA is only adapted to measure in reflection, an optical circulator was used to transform transmission into reflection. The variable coupler was set to $k=0.24$. The attenuation in the ring was swept by the use of a motorized variable attenuator.

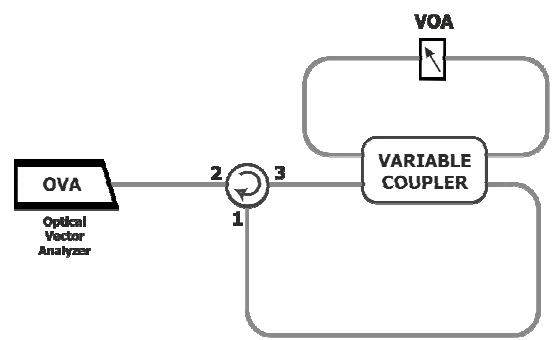

Fig. 3: Experimental setup. VOA: variable optical attenuator

Figure 4 shows two sample group delay spectra obtained for different attenuation values. The central wavelength for the analysis was set at $\sim 1550 \mathrm{~nm}$ with $1 \mathrm{GHz}$ measuring span. The separation between peaks (Fig. 3(a) and (b)) corresponds to $\sim 70 \mathrm{MHz}$ which confirm the $\sim 3 \mathrm{~m}$ loop length. In Figure 4(a), the attenuation was set at $2 \mathrm{~dB}$, corresponding to the case $a<\kappa$. As expected from the above analysis, this configuration gives positive group delays (slow-light regime), peaking in the resonances. In Figure 4(b) the attenuation was increased until $13.5 \mathrm{~dB}$ which corresponds to the case $a>\kappa$ (fast light regime). Unlike the response in Fig. 4(a), the peaks show a negative value ( -9 ns), corresponding to the expected fast-light response.

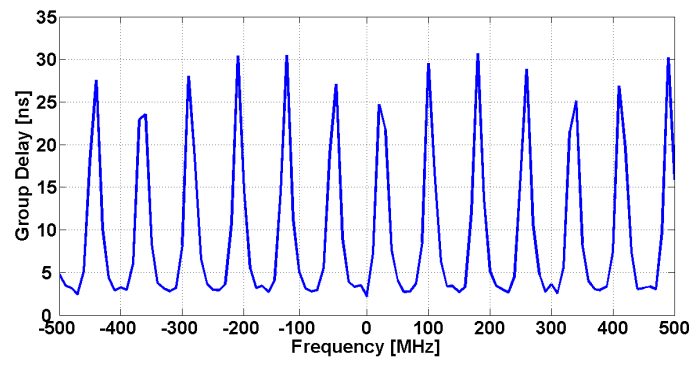

(a)

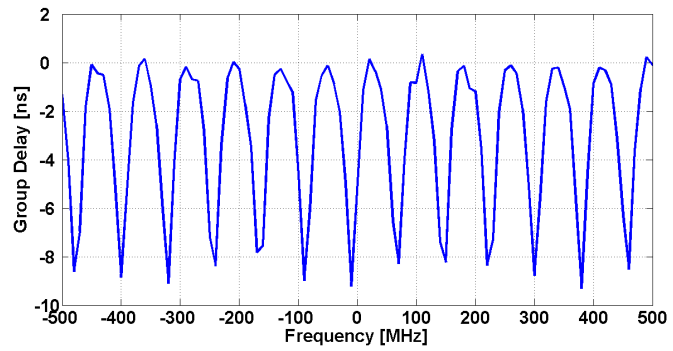

(b)

Fig. 4: Group delay vs. frequency for different attenuation values within the resonator: (a) $2 \mathrm{~dB}$; (b) $13.5 \mathrm{~dB}$.

Figure 5 depicts the good agreement between the results obtained with the experimental setup and the theoretical model explained in section 2. The experimental value of group delay in the resonances is represented for different attenuation values. As it can be seen, a proper agreement is visible between both traces. At critical coupling ( $6 \mathrm{~dB}$ attenuation), the transition between positive group delay and negative group delay is visible (slow-fast-light switch). This critical point and the ones very close to it could not be experimentally resolved due to the extreme frequency resolution required (these points show very large phase sensitivity with respect to frequency, as corresponds to regions of high group index). 


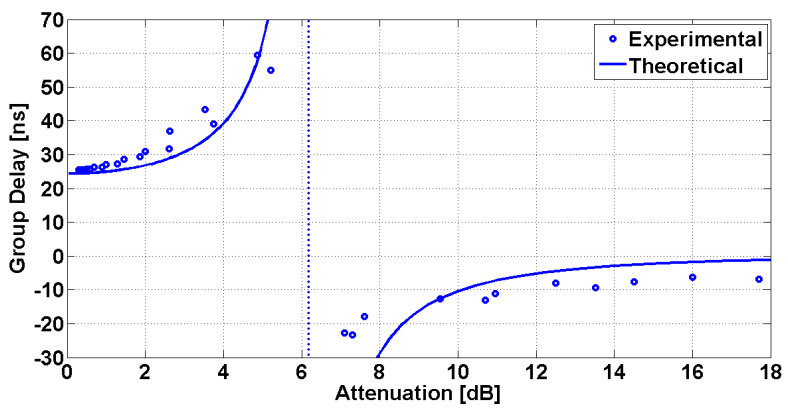

Fig. 5: Mean value of the group delay measured in the resonances for different attenuation values. Experimental and theoretical results are compared showing a proper agreement.

\section{CONCLUSIONS}

We have theoretically and experimentally demonstrated wide-range tunability of the group delay in a lossy fiber ring resonator. The group delay in the device is easily tuned by changing the attenuation and/or coupling ratio in the ring. The setup is capable of switching between positive and negative group delays. This device could be a basic building block in new slow-light-assisted fiber sensors. Moreover, a good agreement between the theory and the developed experiments is demonstrated.

\section{ACKOWLEDGMENTS}

This work was supported in part by the Spanish Ministry of Science and Innovation through projects TEC2009-14423C02-01, TEC2009-14423-C02-02, TEC2010-20224-C02-01 and Innocampus and the Comunidad de Madrid through project FACTOTEM-2. Sonia Martin-Lopez acknowledges funding from the Spanish Ministry of Science and Innovation through a "Juan de la Cierva" contract.

\section{REFERENCES}

[1] J. B. Khurgin and R. S. Tucker, Slow light: Science and applications (CRC Press, Boca Raton, 2009).

[2] M. Soljacic, S. G. Johnson, S. Fan, M. Ibanescu, E. Ippen, and J. D. Joannopoulos, "Photonic-crystal slow-light enhancement of nonlinear phase sensitivity,” J. Opt. Soc. Am. B, vol. 19, 2052, 2002.

[3] C. Monat, B. Corcoran, M. E. Heidari, C. Grillet, B. J. Eggleton, T. P. White, L. O'Faolain and T. F. Krauss, "Slow light enhancement of nonlinear effects in silicon engineered photonic crystal waveguides," Opt. Express, vol. 17, 2944-2953 (2008).

[4] Z. Shi, R. W. Boyd, D. J. Gauthier and C. C. Dudley, "Enhancing the spectral sensitivity of interferometers using slowlight media," Opt. Lett. vol. 32, 915-917 (2007).

[5] M. Terrel, M. J. F. Digonnet and S. Fan, "Performance comparison of slow-light coupled-resonator optical gyroscopes," Laser \& Photon. Rev. vol. 3, 452-465 (2009).

[6] N. A. Mortensen and S. Xiao, "Slow-light enhancement of Beer-Lambert-Bouguer absorption,” Appl. Phys. Lett. vol. 90, 141108 (2007).

[7] S. Chin, I. Dicaire, J. Beugnot, S. Foaleng-Mafang, M. Gonzalez-Herraez, and L. Thévenaz, "Material Slow Light Does Not Enhance Beer-Lambert Absorption," in Slow and Fast Light, OSA Technical Digest (CD) (Optical Society of America, 2009), paper SMA3.

[8] M. Santagiustina, "Electromagnetic Energy Velocity in Slow Light", in Slow and Fast Light, OSA Technical Digest (CD) (Optical Society of America, 2011), paper SLTuB5.

[9] J. Heebner and R. W. Boyd "Slow and fast light in resonator-coupled waveguides" J. Mod. Optics, vol. 49 2629-2636, 2002.

*xabier.angulo@io.cfmac.csic.es; phone +34 915618806 ext.:222 